\title{
Cooling of relativistic electron beams in intense laser pulses: chirps and radiation
}

\author{
S. R. Yoffe*, A. Noble, A. J. Macleod, D. A. Jaroszynski* \\ SCAPA, Department of Physics, SUPA, University of Strathclyde, Glasgow G4 ONG, UK
}

\begin{abstract}
Next-generation high-power laser facilities (such as the Extreme Light Infrastructure) will provide unprecedented field intensities, and will allow us to probe qualitatively new physical regimes for the first time. One of the important fundamental questions which will be addressed is particle dynamics when radiation reaction and quantum effects play a significant role. Classical theories of radiation reaction predict beam cooling in the interaction of a relativistic electron bunch and a high-intensity laser pulse, with final-state properties only dependent on the laser fluence. The observed quantum suppression of this cooling instead exhibits a dependence on the laser intensity directly. This offers the potential for final-state properties to be modified or even controlled by tailoring the intensity profile of the laser pulse. In addition to beam properties, quantum effects will be manifest in the emitted radiation spectra, which could be manipulated for use as radiation sources. We compare predictions made by classical, quasi-classical and stochastic theories of radiation reaction, and investigate the influence of chirped laser pulses on the observed radiation spectra.
\end{abstract}

Keywords: Radiation reaction, quantum effects, beam cooling, chirped laser pulse, radiation spectra, quasi-classical model, stochastic model

\section{Introduction}

The next few years will see a new class of high-intensity laser systems come online, such as the Extreme Light Infrastructure (ELI) [1. The field intensities available at these facilities will far surpass those currently achievable today, and will enable the investigation of physical phenomena not currently accessible. In these regimes, both quantum effects and radiation reaction will dominate the dynamics of charged particles. It is therefore becoming increasingly important to develop a theoretical understanding of these fundamental topics in order to make reliable predictions, but also aid the search for experimental signatures.

Charged particles moving in an electromagnetic field experience the Lorentz force. However, as a charge accelerates it radiates, losing energy and momentum. The selfconsistent inclusion of the effects of this emission in a particle's dynamics, known as radiation reaction, remains a contentious issue. In most current cases, the recoil on the particle due to this radiation emission is negligible compared to the applied forces and is safely neglected. This has prevented experimental observation of radiation reaction, in particular verification of the available theories.

The Lorentz-Abraham-Dirac (LAD) equation [2-4] is the most natural classical description of radiation reac-

\footnotetext{
* Corresponding author

Email addresses: sam.yoffe@strath.ac.uk (S. R. Yoffe),

adam.noble@strath.ac.uk (A. Noble),

alexander.macleod@strath.ac.uk (A. J. Macleod),

d.a.jaroszynski@strath.ac.uk (D. A. Jaroszynski)
}

tion; however, the presence of a third derivative of the position with respect to time leads to various pathologies [5]. There are a number of alternative classical descriptions [5] which only contain (up to) second-order derivatives and are thus not afflicted by these unphysical solutions, but here we focus on one: the Landau-Lifshitz approach [6]. The governing equation is obtained by treating the radiation reaction force as a perturbation to the Lorentz force, and truncating at first order in the 'characteristic time' $\tau:=q^{2} / 6 \pi m\left(\simeq 6 \times 10^{-24} \mathrm{~s}\right.$ for an electron $)$ to give

$$
\ddot{x}^{a}=-\frac{q}{m} F^{a b} \dot{x}_{b}-\tau \frac{q}{m}\left(\dot{F}^{a b} \dot{x}_{b}-\frac{q}{m} \Delta^{a}{ }_{b} F^{b c} F_{c d} \dot{x}^{d}\right)
$$

for a particle of charge $q$ and mass $m$ in an electromagnetic field described by the field tensor $F$. Indices are raised and lowered with the metric tensor $\eta=\operatorname{diag}(-1,1,1,1)$, and repeated indices are summed from 0 to 3 . An overdot denotes differentiation with respect to proper time. The $\dot{x}$-orthogonal projection $\Delta^{a}{ }_{b}:=\delta_{b}^{a}+\dot{x}^{a} \dot{x}_{b}$ ensures that $\ddot{x}$ is orthogonal to $\dot{x}$ and the mass-shell condition is preserved [7. Note that Heaviside-Lorentz units are used with $c=1$.

The Landau-Lifshitz equation (1) has found widespread application, both analytically [8] and numerically in PIC codes [11, 12]. Despite worries regarding its perturbative nature, there is evidence to suggest that it is valid provided only that quantum effects can be neglected [13, 14. However, in order to make predictions for experiments under the extreme conditions expected at ELI, it is essential to capture and describe the relevant quantum effects.

In an attempt to model weakly-quantum effects, we consider a quasi-classical extension to the Landau-Lifshitz 
theory [15. As quantum effects become more important, classical theories overestimate the amount of radiation emitted [16, 7] which is accounted for by a rescaling of the characteristic time $\tau \rightarrow \tau g(\chi)$ in equation (1), where the quantum nonlinearity parameter $\chi=e \hbar \sqrt{F^{a b} F_{a c} \dot{x}_{b} \dot{x}^{c}} / m_{e}^{2}$ and we use the approximation $g(\chi)=\left(1+12 \chi+31 \chi^{2}+\right.$ $\left.3.7 \chi^{3}\right)^{-4 / 9}$ [17]. The radiation reaction force is therefore reduced as $\chi$ increases, compared to the purely classical theory corresponding to $g(\chi)=1$ as $\chi \rightarrow 0$.

Parameters obtainable at ELI are expected to approach $\chi \sim 0.8$, at which point quantum effects can no longer be considered to be weak, and it is unclear whether the quasiclassical theory is at all applicable or if a fully-quantum treatment is necessary [18, 19]. As such, we introduce a stochastic model based on differential single-photonemission probabilities calculated from strong-field quantum electrodynamics (QED) 20 22]. The particle propagates according to the Lorentz force, and at each step the probability of emitting a photon is evaluated and compared to a random number in order to determine whether or not emission occurs. If so, a photon energy is determined and the particle's momentum updated accordingly.

Recently, an analytical solution to the Vlasov equation was found for radiation reaction according to the LandauLifshitz theory [16, and used to demonstrate how the inclusion of quantum effects using the quasi-classical model causes the observed beam cooling to become anisotropic 23. A model for linearly-chirped laser pulses was used to study electron beam cooling using the quasi-classical theory for radiation reaction [7. This present work builds on both of these investigations, extending the analysis to circular polarisation and introducing a stochastic model for single-photon emission before looking at the influence of a linear frequency chirp on the measured radiation spectrum.

\section{Quasi-classical beam cooling}

In a plane wave, the only dependence on spacetime of the electromagnetic field tensor $F$ is through the phase $\phi=-k \cdot x=\omega t-\vec{k} \cdot \vec{x}$, where $k=(\omega, \vec{k})$ is the (null) laser wavevector. For an arbitrarily-polarised plane-wave laser, the field tensor can be expressed

$$
\frac{q}{m} F_{b}^{a}=a_{\epsilon}(\phi)\left(\epsilon^{a} k_{b}-k^{a} \epsilon_{b}\right)+a_{\lambda}(\phi)\left(\lambda^{a} k_{b}-k^{a} \lambda_{b}\right),
$$

with $\epsilon, \lambda$ the mutually-orthogonal (transverse) polarisation directions and $a_{i}(\phi)$ dimensionless measures of the electric field strength in the direction $i \in\{\epsilon, \lambda\}$. We consider $a_{i}(\phi)=a_{\text {env }}(\phi) \psi_{i}(\phi)$, with the oscillation $\psi_{i}(\phi)$ modulated by a $\sin ^{2}$-envelope

$$
a_{\mathrm{env}}(\phi)=a_{0} \sin ^{2}(\pi \phi / L) \quad \text { for } \quad 0<\phi<L
$$

and zero otherwise, where $L=2 \pi N$ is the "length" of a pulse containing $N$ cycles. For a representative wavelength
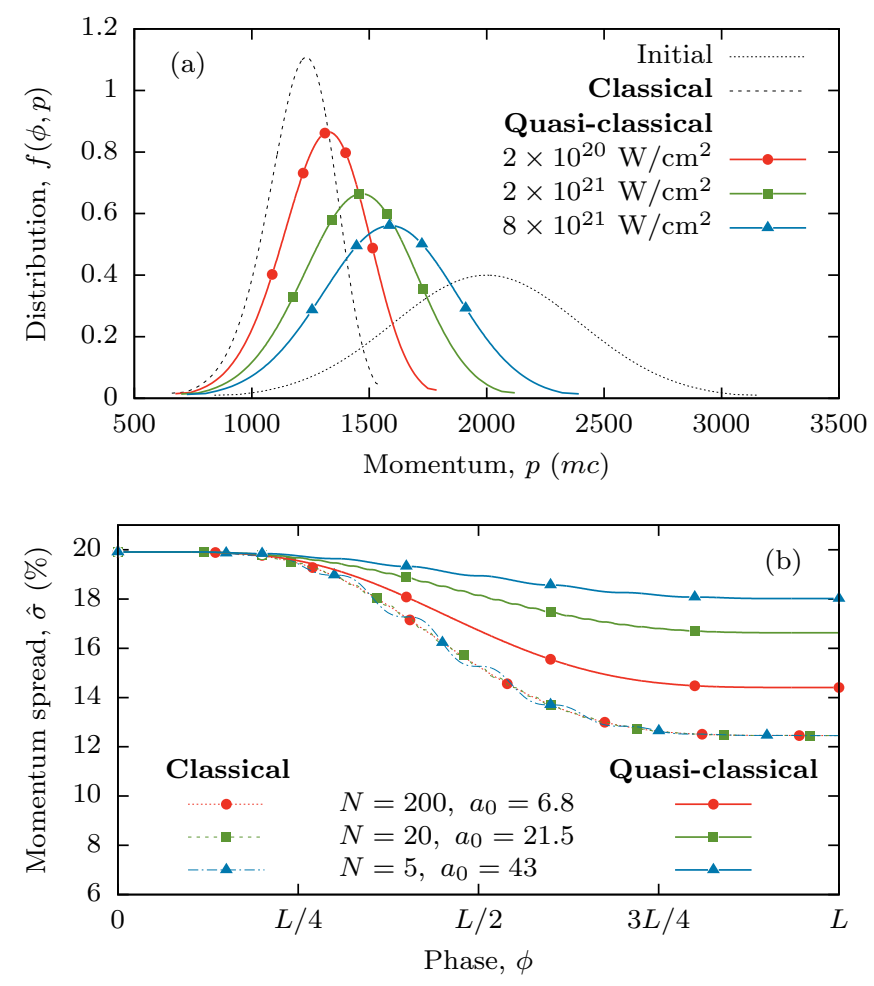

Figure 1: (Colour online.) Predictions for the (a) final-state particle distribution and (b) evolution of the relative momentum spread using the classical (dashed) and quasi-classical (solid) theories. The increase in intensity (reduction in $N$ ) reduces the amount of quasiclassical beam cooling observed.

of $\lambda=800 \mathrm{~nm}$, this corresponds to a pulse with full-width half-maximum (FWHM) duration $T=N \lambda / 2 c=1.33 N$ fs.

In the following, the initial electron bunch is taken to be Gaussian-distributed with a $20 \%$ spread around a central Lorentz $\gamma$-factor of $\gamma_{0}=2000$, corresponding to an energy of around $1 \mathrm{GeV}$. This bunch collides head on with a counterpropagating laser pulse. Our exact numerical methods are described in detail elsewhere [16, 7].

\subsection{Linear polarisation}

For a plane-wave laser, linearly polarised in the $\epsilon$ direction, we have the oscillating functions $\psi_{\epsilon}(\phi)=\sin (\phi)$ and $\psi_{\lambda}(\phi)=0$. Figure 1 shows the interaction of the initial Gaussian beam with pulses ranging from $N=5$ to 200 cycles. Since the final-state predictions of the classical Landau-Lifshitz theory only depend on the fluence of the pulse [24], $\mathcal{E} \propto N a_{0}^{2}$, we choose $a_{0}$ such that we maintain $N a_{0}^{2}=9248$ (corresponding to a peak intensity $I=2 \times 10^{21} \mathrm{~W} / \mathrm{cm}^{2}$ for $\lambda=800 \mathrm{~nm}$ and $T=27 \mathrm{fs}$ ) and the classical final-state properties all agree. This can be seen explicitly in Fig. 1(b) for the relative momentum spread, $\hat{\sigma}=\sigma / \bar{p}$, where $\sigma$ is the standard deviation and $\bar{p}$ is the mean momentum. Although the measurement does not agree during the evolution due to differences in the energy distribution caused by the differing numbers of cycles, after the pulses have passed the predictions coincide. The final-state particle distribution can be seen in Fig. 1(a) 


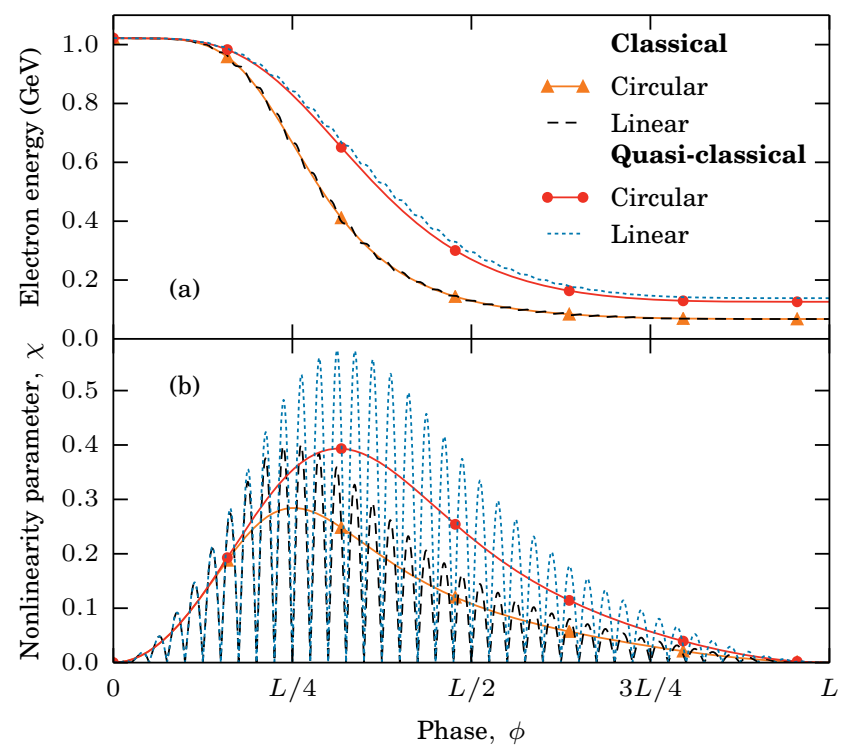

Figure 2: (Colour online.) Evolution of the (a) electron energy and (b) nonlinearity parameter predicted by the classical and quasiclassical theories, for both linear and circular polarisation. The quasi-classical theory predicts higher values of $\chi$ and, unlike the classical case, a dependence of the final energy on the polarisation.

to become more tightly distributed around a lower mean momentum compared to the initial Gaussian distribution (the beam has cooled and lost energy) but again the result is identical provided the fluence is kept constant.

By contrast, the quasi-classical predictions are sensitive to the intensity directly [16]. As the intensity is increased, $\chi$ experienced by the electrons increases, which in turn acts to suppress radiation reaction. As such, we observe less energy loss and less cooling of the electron beam. The cooling is also found to become anisotropic [23, 16].

\subsection{Circular polarisation}

Since the quasi-classical predictions depend on the distribution of energy within the pulse, we can consider the effect of instead using a circularly-polarised laser. In this case, the magnitude of the field experienced by the electron does not oscillate as in the linear case, but rather a much smoother profile is encountered. As such, the peak intensity observed is half that for the linear case. (They have the same cycle-averaged intensity, and the same fluence.) The transverse components of the field oscillate with $\psi_{\epsilon}(\phi)=\sin (\phi) / \sqrt{2}$ and $\psi_{\lambda}(\phi)=\cos (\phi) / \sqrt{2}$.

The predictions made by the classical and quasi-classical theories are shown in Fig. 2 for $N=20$ cycles and $a_{0}=$ 100 , corresponding to peak intensities $I_{\text {lin }}=4.28 \times 10^{22}$ and $I_{\text {circ }}=2.14 \times 10^{22} \mathrm{~W} / \mathrm{cm}^{2}$. Once again, because the fluence contained by the two pulses is unchanged, the classical theory predicts identical final-state properties in Fig. 2(a). By contrast, the difference in the nonlinearity parameter experienced by the electron, as shown in Fig. 2(b), leads to a small difference in the final-state quasi-classical predictions in Fig. 2(a).
In both Figs. 2(a) and 2(b), during the evolution the response to the circularly-polarised pulse is observed to vary smoothly and not display fluctuations on the scale of the wavelength like the linearly-polarised pulse.

\section{Stochastic photon emission}

\subsection{Comparison of stochastic and quasi-classical models}

The photon emission model discussed at the end of section 1 explicitly captures the stochastic nature of photon emission, and as such should remain valid beyond the quasi-classical theory. It can therefore be used to gauge the performance of the quasi-classical theory as the quantum nonlinearity parameter increases. This is useful, since obtaining quasi-classical predictions requires significantly less computing power. This is in part due to the fact that a large ensemble is required to extract average predictions from the stochastic theory.

Figure 3 (a) shows the evolution of the energy for a single electron with initial $\gamma_{0}=2000$ colliding with a 10 cycle, circularly-polarised pulse with $a_{0}=100$ according to the classical and quasi-classical theories. An ensemble of 15000 identical electrons was propagated using the stochastic theory, of which the trajectory of one (random) particle is also plotted. The trajectory exhibits discontinuous jumps of various sizes as the electron emits photons of different energies. However, the average trajectory of all electrons in the ensemble shows remarkable agreement with the quasi-classical prediction, despite the value of the nonlinearity parameter $\chi>0.5$ shown in Fig. 3 (b), far beyond the expected region of validity for the quasi-classical

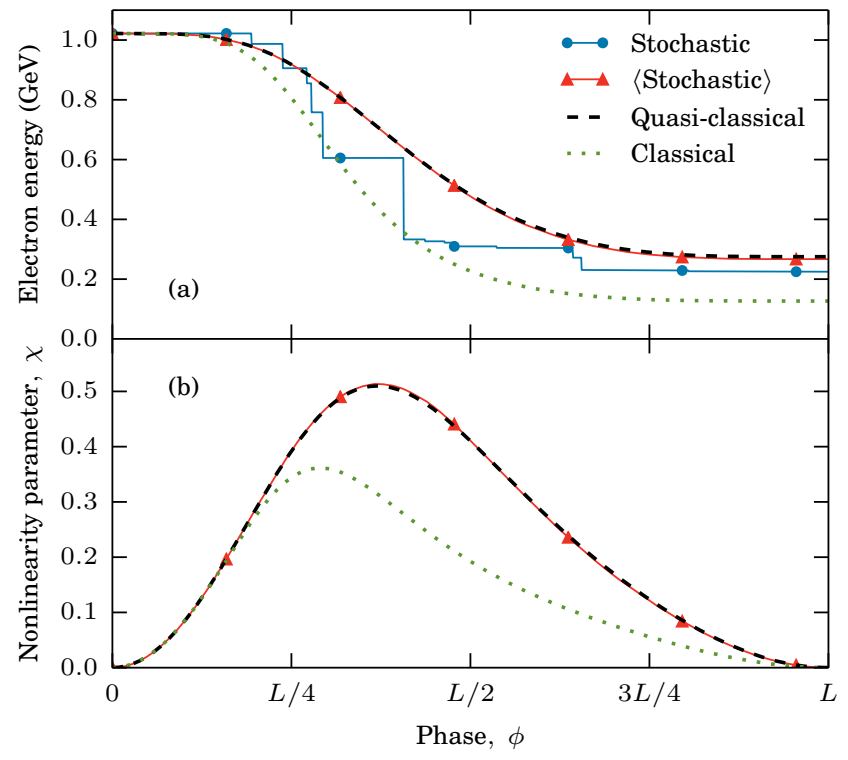

Figure 3: (Colour online.) Comparison of the (a) electron energy and (b) nonlinearity parameter in the classical, quasi-classical, and stochastic theories. The stochastic trajectory in (a) shows discontinuous drops in energy, while the classical theory overestimates the energy lost by the electron. The ensemble-averaged stochastic predictions agree well with the quasi-classical theory. 

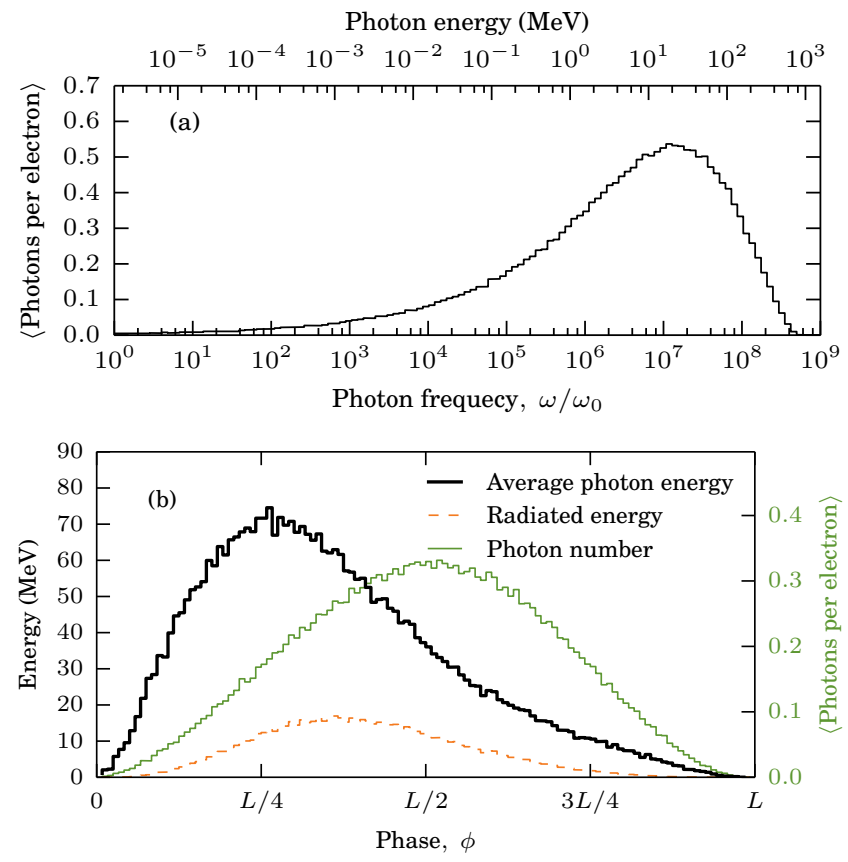

Figure 4: (Colour online.) The (a) emitted photon spectrum and (b) average emitted photon energy as a function of emission position within the pulse. The dashed curve in (b) gives the total energy radiated at each position, which is used to calculate the positivelyskewed average photon energy (thick solid line).

theory, $\chi^{2} \ll 1$. This unexpected result could potentially save large amounts of computation.

\subsection{Radiation spectra}

An immediate consequence of the stochastic model is that one has direct access to the spectrum of the radiation emitted by an electron as it navigates the laser pulse. Our ensemble of 15000 electrons produced 299412 photons of various energies (assumed to be perfectly backscattered), from which we generate the graph shown in Fig. 4(a). The spectrum is compatible with a $2 \gamma^{2} \simeq 10^{7}$ upshift of the laser fundamental frequency, $\omega_{0}$. We also know where each emission happened, allowing the photon number and total emitted energy to be found as a function of the location (phase) within the pulse in Fig. 4(b). The average emitted photon energy (as a function of the emission phase) can then be extracted. We note that the photon number is essentially proportional to the (symmetric) $\sin ^{2}(\pi \phi / L)$ intensity envelope (3), while photons radiated towards the beginning of the interaction have higher average energy than those emitted towards the middle or end, with a peak around $70 \mathrm{MeV}$ at $\phi \simeq L / 4$ (earlier than the peak in $\chi$ at $\phi \simeq L / 3$ from Fig. 3(b)). This is because the emitted photon energy depends in part on the electron energy, which is decreasing with every emission.

\subsection{Chirped laser pulses}

One of the methods used to reach ultra-high intensities is chirped pulse amplification (CPA). As such, the result- ing laser pulses with which many experiments will be carried out may contain a frequency chirp. Moreover, there are often practical benefits to deliberately introducing a frequency chirp 25 27. Their impact on beam dynamics and emitted radiation is therefore directly relevant for future high-intensity experiments. The influence of chirped pulses on beam dynamics has been previously investigated using linearly-polarised pulses [7, and an intuitive explanation was provided for the observed effect. Here, we use the stochastic quantum model and investigate the changes to the emitted radiation spectrum.

There have been several recent investigations in which a linear frequency chirp is defined 25] 28, including an interesting study on the radiation spectra predicted by the classical Landau-Lifshitz theory [27] where they found that negative chirps led to an increased rate of electron energy loss and a stronger peak in the spectrum, as well as an increase in the radiated frequencies. We follow the chirping formulation presented in Reference [7, in which the number of cycles contained by the pulse is kept the same such that the chirped length becomes $L_{\Delta}=2 \pi N /(1+\Delta / 2)$ for the chirp rate $\Delta$. The linearly-chirped phase is

$$
\eta(\phi ; \Delta)=\phi\left(1+\phi \Delta / 2 L_{\Delta}\right)
$$

and the dimensionless fields are written

$$
a_{i}(\phi ; \Delta)=A_{\mathrm{env}}(\phi ; \Delta) \Psi_{i}(\phi ; \Delta)
$$

where the oscillation functions and envelope are given by

$$
\begin{aligned}
\Psi_{i}(\phi ; \Delta) & =\psi_{i}(\eta(\phi ; \Delta)) \quad \text { and } \\
A_{\mathrm{env}}(\phi ; \Delta) & =a_{\mathrm{env}}(\phi) \sqrt{1+\Delta / 2},
\end{aligned}
$$

respectively, for $0<\phi<L_{\Delta}$ (otherwise zero). The factor $\sqrt{1+\Delta / 2}$ is included to ensure that the chirped pulses all contain the same fluence.

In a similar manner to Fig. 4. Fig. 5(a) shows the spectrum and Fig. 5(b) the average emitted photon energy as a function of emission phase within the pulse, plotted for (large) chirps $\Delta= \pm 0.5$, along with the unchirped pulse $(\Delta=0)$. Statistics were obtained from 352065 (263031) photons for the negative (positive) chirp. Figure 5(a) shows that the average emitted photon number does increase (decrease) for the negative (positive) chirp, but Fig. 5(b) shows a corresponding drop (rise) in the average photon energy. Together, these do not necessarily correspond to increased energy emitted with the negative chirp. The dashed line therefore shows the cumulative energy radiated as the electron moves through the pulse, confirming that the negative chirp does cause an increase in radiation, despite the lower average photon energy. It is also interesting to note that, according to the stochastic theory, the chirp rate does not affect the spectral cut-off, unlike the classical case [27. In fact, a slight decrease in the peak frequency is observed for the negative chirp. 


\section{Conclusions}

The availability of high-powered lasers in the next few years will drive experimental physics into unchartered waters previously inaccessible, where quantum effects will have a dominant role. It is crucial to develop an understanding of the fundamental processes involved in order to both make predictions and guide experiment design, but also explain experimental findings.

In this paper, we have investigated the importance of quantum effects in predictions of radiation reaction in the interaction between a high-intensity laser pulse and a highly-relativistic electron beam. Beam cooling is observed to be reduced in quasi-classical predictions as quantum effects become important, leading to a direct dependence on the laser intensity profile. This has been modified using linear and circular polarisation, resulting in a small effect, and the introduction of a linear frequency chirp.

A stochastic model based on single photon emission has been compared to quasi-classical predictions with excellent agreement for $\chi \simeq 0.5$, provided an ensemble of electrons is used. A negative chirp is found to increase the electron energy loss and cause increased photon emission, albeit with a reduced average emitted photon energy. Unlike classical predictions 27, the stochastic model does not produce a significant change in the maximum radiated frequency.
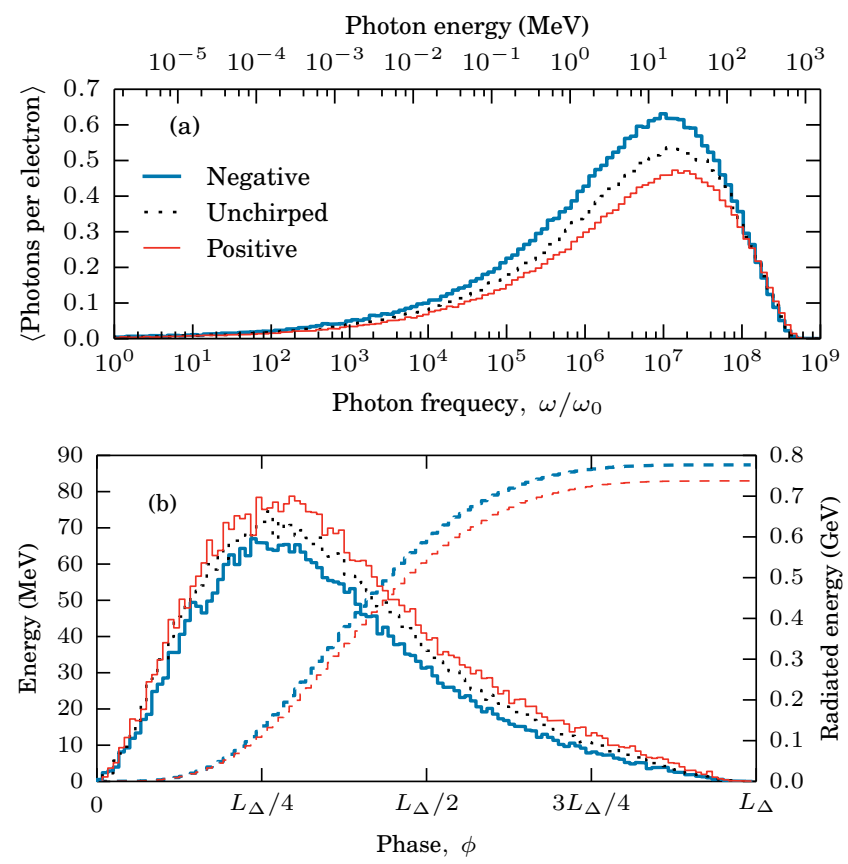

Figure 5: (Colour online.) The (a) emitted radiation spectra and (b) average emitted photon energy, with positively- and negativelychirped pulses. The thick (thin) curves are for the negative (positive) chirp. The dashed curves in (b) are the cumulative energy radiated.

\section{Acknowledgements}

We thank Chris Harvey and Amol Holkundkar for helpful discussion. This work is supported by the UK EPSRC (Grant EP/J018171/1); the ELI-NP Project; the Cockcroft Institute; and the European Commission FP7 projects Laserlab-Europe (Grant 284464) and EuCARD-2 (Grant 312453). Datasets available online [29, 30].

\section{References}

[1] http://www.eli-laser.eu/; http://www.eli-np.ro/

[2] H. A. Lorentz, The Theory of Electrons and its Applications to the Phenomena of Light and Radiant Heat, Stechert, New York, 1916.

[3] M. Abraham, The Classical Theory of Electricity and Magnetism, Blackie, London, 1932.

[4] P. A. M. Dirac, Classical Theory of Radiating Electrons, Proc. R. Soc. A 167 (1938) 148.

[5] D. A. Burton, A. Noble, Aspects of electromagnetic radiation reaction in strong fields, Contemporary Physics 55 (2014) 110.

[6] L. D. Landau, E. M. Lifshitz, The Classical Theory of Fields, Pergamon, London, 1962.

[7] S. R. Yoffe, A. Noble, Y. Kravets, D. A. Jaroszynski, Cooling of relativistic electron beams in chirped laser pulses, Proc. SPIE 9509 (2015) 950905.

[8] C. Harvey, T. Heinzl, M. Marklund, Symmetry breaking from radiation reaction in ultra-intense laser fields, Phys. Rev. D 84 (2011) 116005.

[9] G. Lehmann, K. H. Spatschek, Energy gain of an electron by a laser pulse in the presence of radiation reaction, Phys. Rev. E 84 (2011) 046409

[10] Y. Hadad, L. Labun, J. Rafelski, N. Elkina, C. Klier, H. Ruhl, Effects of radiation reaction in relativistic laser acceleration, Phys. Rev. D 82 (2010) 096012.

[11] M. Vranic, J. L. Martins, J. Vieira, R. A. Fonseca, L. O. Silva, All-optical radiation reaction at $10^{21} \mathrm{~W} / \mathrm{cm}^{2}$, Phys. Rev. Lett. 113 (2014) 134801.

[12] M. Vranic, J. L. Martins, R. A. Fonseca, L. O. Silva, Classical Radiation Reaction in Particle-In-Cell Simulations, arXiv (2015) 1502.02432.

[13] Y. Kravets, A. Noble, D. A. Jaroszynski, Radiation reaction effects on the interaction of an electron with an intense laser pulse, Phys. Rev. E 88 (2013) 011201(R).

[14] H. Spohn, The critical manifold of the Lorentz-Dirac equation, Europhys. Lett. 50 (2000) 287.

[15] J. G. Kirk, A. R. Bell, I. Arka, Pair production in counterpropagating laser beams, Plasma Phys. Control. Fusion 51 (2009) 085008.

[16] S. R. Yoffe, Y. Kravets, A. Noble, D. A. Jaroszynski, Longitudinal and transverse cooling of relativistic electron beams in intense laser pulses, New J. Phys. 17 (2015) 053025.

[17] A. G. R. Thomas, C. P. Ridgers, S. S. Bulanov, B. J. Griffin, S. P. D. Mangles, Strong Radiation-Damping Effects in a Gamma-Ray Source Generated by the Interaction of a HighIntensity Laser with a Wakefield-Accelerated Electron Beam, Phys. Rev. X 2 (2012) 041004.

[18] A. Di Piazza, K. Z. Hatsagortsyan, C. H. Keitel, Quantum Radiation Reaction Effects in Multiphoton Compton Scattering, Phys. Rev. Lett. 105 (2010) 220403.

[19] A. Ilderton, G. Torgrimsson, Radiation reaction in strong field QED, Phys. Lett. B 725 (2013) 481.

[20] V. I. Ritus, Quantum effects of the interaction of elementary particles with an intense electromagnetic field, J. Sov. Laser Res. 6 (1985) 497.

[21] D. G. Green, C. N. Harvey, Transverse Spreading of Electrons in High-Intensity Laser Fields, Phys. Rev. Lett. 112 (2014) 164801.

[22] T. G. Blackburn, C. P. Ridgers, J. G. Kirk, A. R. Bell, Quantum Radiation Reaction in Laser-Electron-Beam Collisions, Phys. Rev. Lett. 112 (2014) 015001. 
[23] A. Noble, S. R. Yoffe, D. A. Jaroszynski, Anisotropic cooling of electron beams interacting with intense laser pulses, CLF Annual Report 2014/2015.

[24] N. Neitz, A. Di Piazza, Electron-beam dynamics in a strong laser field including quantum radiation reaction, Phys. Rev. A 90 (2014) 022102

[25] I. Ghebregziabher, B. A. Shadwick, D. Umstadter, Spectral bandwidth reduction of Thomson scattered light by pulse chirping, Phys. Rev. Spec. Top. Accel. Beams 16 (2013) 030705.

[26] S. G. Rykovanov, C. G. R. Geddes, C. B. Schroeder, E. Esarey, W. P. Leemans, Controlling the spectral shape of nonlinear Thomson scattering with proper laser chirping, arXiv (2014) 1412.2517 .

[27] A. R. Holkundkar, C. N. Harvey, M. Mattias, Thomson scattering in high-intensity chirped laser pulses, Phys. Plasmas 22 (2015) 103103.

[28] E. Yazdani, R. Sadighi-Bonabi, H. Afarideh, Z. Riazi, H. Hora, Electron heating enhancement by frequency-chirped laser pulses, J. Appl. Phys. 116 (2014) 103302.

[29] S. R. Yoffe, A. Noble, Y. Kravets, D. A. Jaroszynski (2015). doi:10.15129/79f9c58d-7a43-4cc0-a613-ebc028519e5b

[30] S. R. Yoffe, A. Noble, A. J. Macleod, D. A. Jaroszynski (2015). doi:10.15129/f478bdb8-24aa-4f1a-ac21-2e6c5a55946a 